\title{
Stabilization of a cold cathode electron beam glow discharge for surface treatment
}

\author{
N. Mingolo and C. R. González \\ Lab. de Haces Dirigidos, Depto. de Física, Facultad de Ingeniería, Universidad de Buenos Aires, \\ Paseo Colón 850, 1063 Buenos Aires, Argentina \\ O. E. Martínez \\ Lab. de Electrónica Cuántica, Depto. de Física, Universidad de Buenos Aires, Pabellón 1, \\ Ciudad Universitaria, 1428 Buenos Aires, Argentina \\ J. J. Rocca \\ Department of Electrical Engineering, Colorado State University, Fort Collins, Colorado 80523
}

(Received 27 February 1997; accepted for publication 3 June 1997)

We have demonstrated that the reproducibility of electron beam pulses generated by a high power, cold cathode glow discharge is greatly improved by adding a small continuous keep-alive discharge current. A current of the order of $200 \mu \mathrm{A}$ was found to limit the shot to shot current variation to within $1.5 \%$. This stabilization in turn reduces by an order of magnitude the fluctuations of the energy density deposited on the target, demonstrating a reliable energy source for surface treatment.

(C) 1997 American Institute of Physics. [S0021-8979(97)05217-1]

Simple cold cathode high voltage glow discharges can generate powerful electron beams for materials processing. ${ }^{1-6}$ Of particular interest is the deposition of high power densities for surface treatment of metallic surfaces. ${ }^{6,7}$ We have previously reported the development of an electron gun of simple implementation that provides nearly monoenergetic electron pulses for this purpose. ${ }^{6,7}$ The cold cathode electron gun used in the experiments described herein, which operates at voltages between 10 and $30 \mathrm{kV}$, generates pulses with adjustable pulse widths between 10 and $30 \mu$ s and currents up to $120 \mathrm{~A}$. The Lorentz force generated by the interaction of the electron flow and its self-generated magnetic field focuses the beam, allowing for energy density depositions of up to $40 \mathrm{~J} / \mathrm{cm}^{2}$ in a $\sim 1 \mathrm{~cm}^{2}$ spot area. This energy density can melt metallic surfaces placed in the focal region of the beam. ${ }^{6}$

The depth of the melted surface depends on the electron penetration and on the heat diffusion. The rapid cooling of the surface layer of the sample by heat conduction modifies the surface microstructure. However, a limitation arises in this application from the fluctuations in the beam current from one shot to another, not only changing the electron beam power but also the spot size of the beam at the sample. This can give rise to large shot to shot fluctuations in the surface treatment even for small changes in the peak current, as recently shown by Etcheverry et al. $^{7}$ In addition, sporadic arcs are observed that can disrupt the high impedance glow discharge mode of operation necessary for the generation of the electron beam, further complicating the reliable treatment of materials surfaces.

In this communication we show that the addition of a small continuous current discharge $(<1 \mathrm{~mA})$ greatly stabilizes the electron beam generation. In the experiments we conducted this "keep-alive" current reduced the shot to shot current fluctuations to within $1.5 \%$. This in turn reduces by an order of magnitude the fluctuation of the spot size of the beam on the target to an acceptable $11 \%$, demonstrating a reliable electron source for surface treatment.

The high voltage cold cathode helium glow discharge used to generate the electron beam is schematically illustrated in Fig. 1. It consists of a planar aluminum cathode 7.5 $\mathrm{cm}$ in diameter surrounded by an insulator shield, and an anode, both enclosed in a glass tube. The tube is evacuated and helium is continuously flown to maintain a typical operating pressure of 300 mTorr. A small amount of $\mathrm{O}_{2}$ is also added to maintain the cathode surface oxidized with the purposes of increasing the secondary electron emission yield and reducing sputtering. ${ }^{5}$ The pulsed electron beam is generated by discharging a negatively charged capacitor between the cathode and a grounded anode by means of a triggered spark-gap switch. The discharge is maintained by electrons emitted from the cathode surface by the bombardment of ions and fast neutral atoms. The majority of the discharge voltage falls within about $1 \mathrm{~cm}$ from the cathode surface in the cathode sheath region formed by the accumulation of positive space charge. ${ }^{8}$ This space charge acts as a virtual anode for the emitted electrons, making the position of the anode electrode (sample) rather irrelevant for practical purposes. The large electric field present in the cathode sheath region rapidly accelerates the electrons to form an energetic electron beam. The beam self-focused due to the Lorentz force created by its own magnetic field. ${ }^{7}$

The dc keep-alive current was created by incorporating in the discharge circuit a resistance ( $R 2$ in Fig. 1$)$ that bypasses the spark-gap switch. The value of the resistor was selected in order to maintain a continuous glow discharge while avoiding overheating of the sample. For the usual operating helium pressure mentioned above a stable keep-alive current of less than $1 \mathrm{~mA}$ is obtained at a cathode voltage of 400-500 V.

In order to determine the improvements obtained by the keep-alive current the evolution of the discharge voltage was measured for series of shots with and without the keepalive current. The corresponding discharge current was determined from the rate of change of the charge on the capacitor $(I=C d V / d t)$. Figures 2(a) and 2(b) show the current pulse corresponding to five consecutive discharge shots without and with a keep-alive current of $200 \mu \mathrm{A}$, respectively. 


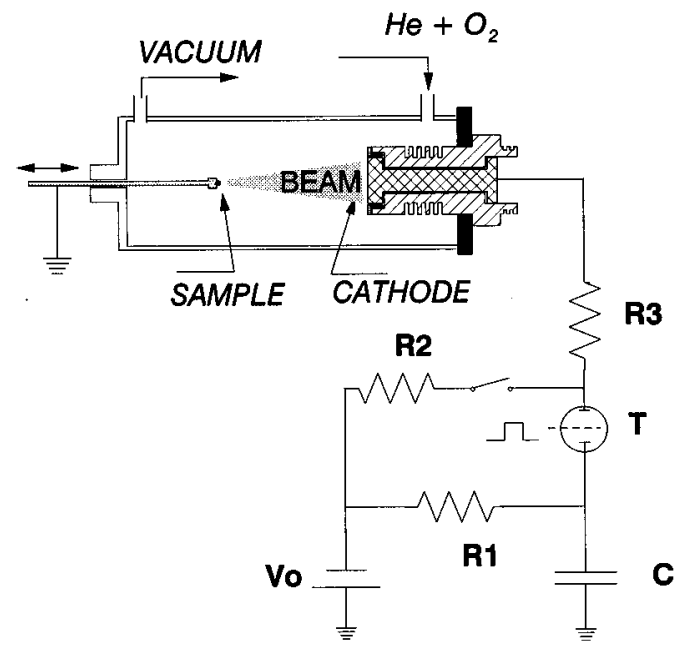

FIG. 1. Schematic diagram of the electron gun and discharge circuit. The discharge and sample chamber is kept at a He pressure of about $300 \mathrm{mTorr}$ with a small partial pressure of $\mathrm{O}_{2}$ of about 10 mTorr. The capacitor $C=90 \mathrm{nF}$ is charged through resistor $R_{1}=20 \mathrm{M} \Omega$, and is unloaded through the gun and the ballast $R_{3}=25 \Omega$, when the spark-gap is triggered ( $T$ ). The beam self-focuses due to the Lorentz force and the position of the sample determines the treated beam area. A keep-alive current is added bypassing the spark-gap with a high impedance resistor $R_{2}=100 \mathrm{M} \Omega$, yielding a current of about $200 \mu \mathrm{A}$ and a cathode voltage of $400 \mathrm{~V}$.

The corresponding measured voltage curves are shown as inserts. The data were obtained charging the capacitor to 18 $\mathrm{kV}$, and setting the pressure $\left(\mathrm{He}+\mathrm{O}_{2}\right)$ to 290 mTorr. A very significant improvement in the shot to shot reproducibility of the discharge parameters is observed to result from the use of the keep-alive current, reducing the current fluctuations from more than $10 \%$ to $1.5 \%$. Moreover, the keep-alive current is observed to increase the discharge current by more than a factor of 2 , and to very significantly reduce the number of arcs observed.

The consequences of this current stabilization in the use of this electron beam in surface treatment can be quantitatively evaluated using the simple semiempirical model of Etcheverry et al. ${ }^{7}$ To describe the dependence of the current on the discharge parameters this model makes use of the empirical relation:

$$
I=a V^{k} P^{m},
$$

with $k=3$ and $m=2.2$, where $V$ is the cathode voltage and $P$ the He partial pressure, expressed in $\mathrm{kV}$ and mTorr, respectively. The parameter $a$ depends on the value of the ballast resistor ( $R 3$ in Fig. 1) and the condition of the cathode surface. Therefore it also depends on the oxygen partial pressure and on the repetition rate.

Using this model the beam area $S$ at a sample located at a distance $d$ from the cathode is determined to be

$$
S=\pi R_{0}^{2}\left(1-\frac{\mu_{0}}{4 \pi} \frac{I}{\sqrt{V}} \sqrt{\frac{e}{2 m}} \frac{d^{2}}{R_{0}^{2}}\right)^{2},
$$

where $R_{0}$ is the cathode radius. From Eqs. (1) and (2) the sensitivity of the beam area to fluctuations in the parameter $a$ is given by:
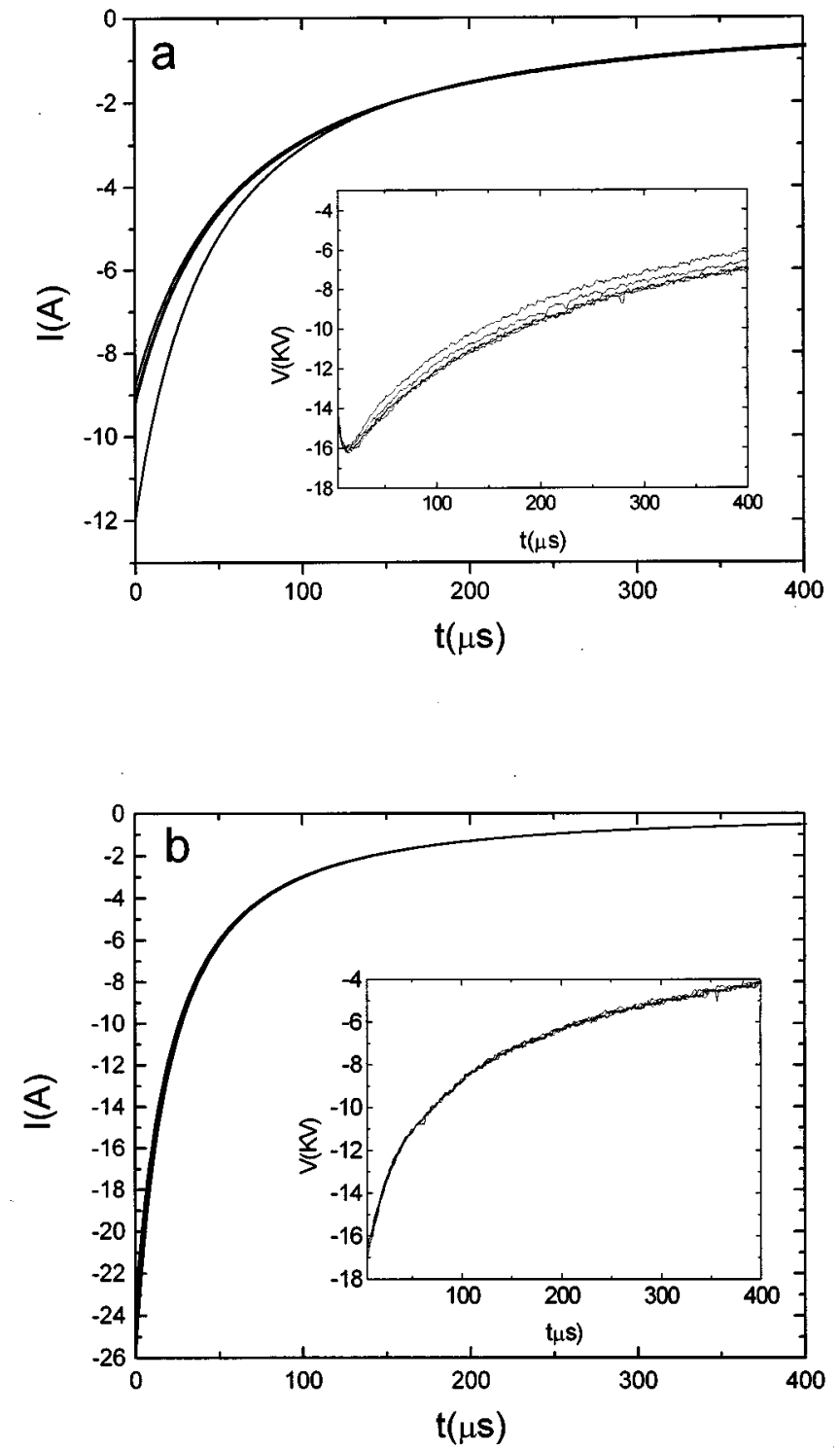

FIG. 2. (a) Current vs time for five discharge shots without keep-alive current. The inset shows the correspondent measured cathode voltage plots. The changes in the voltage time constant give rise to large changes in the current, and even larger fluctuations in the treated area. (b) Same as in (a) but with a $200 \mu \mathrm{A}$ keep-alive current. The five pulses almost overlap and a reduction in the current fluctuations is evident. An increase in the peak current is also noticeable.

$$
\frac{d \ln (S)}{d \ln (a)}=\frac{2 R_{0}}{r},
$$

where $r$ is the beam radius at the sample. This expression shows the extreme sensitivity of the beam area to the parameter $a$, which is larger for strong focusing conditions. For example, for the parameters corresponding to our system, $r$ $\approx 1 \mathrm{~cm}$ and $R_{0}=3.75 \mathrm{~cm}$, a $5 \%$ change in the parameter $a$ results in a $35 \%$ change in the electron beam area.

Figure 3 shows the computed variation of the electron beam size as a function of the distance from the cathode, clearly showing the importance that relatively small current variations have of the size of the treated area, and consequently on the energy density deposition. The results corresponding to three consecutively measured discharge pulses 

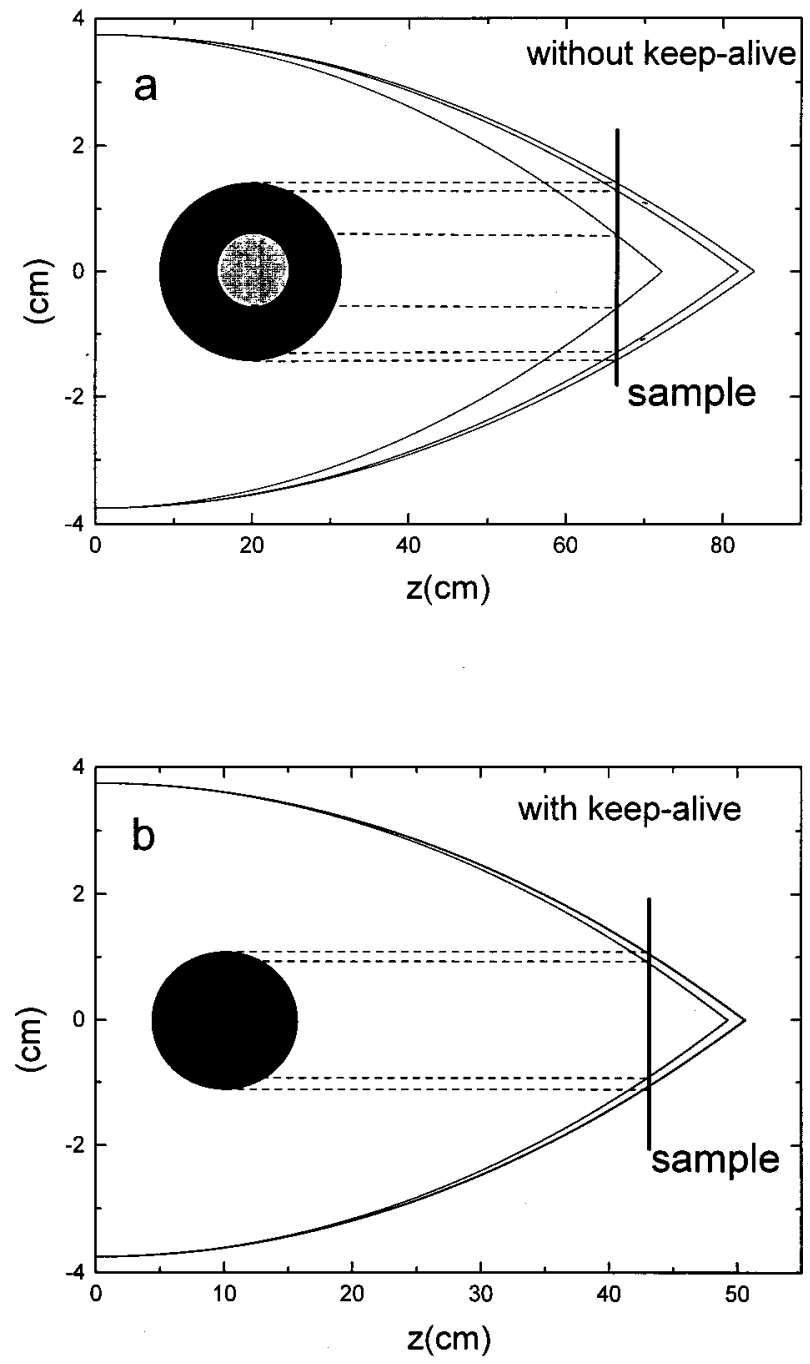

FIG. 3. Electron beam profile as a function of the distance from the cathode for three consecutive discharge pulses computed using data from Fig. 2: (a) shots without keep-alive current, (b) shots with keep-alive current. The treated area depends on the sample location. The circles show the corresponding beam area at the sample. Small changes in the beam current are shown to strongly affect the focusing, and hence the treated area.

obtained without and with the keep-alive current are shown in Figs. 3(a) and 3(b), respectively. The corresponding sizes of the treated areas are also shown for the location of the target sample indicated. As seen in the figure, the origin of the fluctuations in the treated area arises from changes in the self-focusing distance.

To analyze the significance of the current fluctuations measured in our experiment on surface treatment we fitted the voltage data of Fig. 2 with the expression from Ref. 7:

$$
V(t)=V_{0}\left(1+\frac{a P^{m} 2 V_{0}^{2} t}{C}\right)^{-1 / 2}
$$

and we recovered the parameter $a$ from the fit. For the data corresponding to discharges without keep-alive current the fit with Eq. (4) yields a parameter $a=0.026 \pm 0.0035$ (i.e., $13 \%$ fluctuations). If the sample is positioned such that the beam has a radius of about $1 \mathrm{~cm}$ at the target, the resulting fluctuations in the treated area approaches $100 \%$. In contrast, for the data obtained with the keep-alive current the parameter $a$ increases more than twice, to $a=(0.066 \pm 0.001)$, with fluctuations of only $1.5 \%$. This current stabilization is computed to limit the beam area fluctuations in the sample to $11 \%$.

In summary, it was possible to reduce the shot to shot current and beam area fluctuations of a pulse glow discharge electron beam to acceptable values using a small keep-alive current. A keep-alive current of only $200 \mu \mathrm{A}$ reduced the current fluctuations, associated in the semiempirical model of Ref. 7 with fluctuations of the parameter $a$, from $13 \%$ to $1.5 \%$. This shot to shot stabilization yielded an acceptable $11 \%$ fluctuation in the beam area at the target. Moreover, the keep-alive current was also found to stabilize the day to day changes in the discharge current. With the keep-alive current the parameter $a$ was stable and had the same value obtained from a freshly prepared cathode. This suggests that the ion bombardment associated with the small direct current discharge stabilizes the cathode surface. In this manner the current pulses were observed to be practically independent of the number of shots and the discharge repetition rate. In addition, an important reduction of glow-to-arc transitions was observed. In conclusion we have achieved reliable and reproducible operation of a high voltage, cold cathode electron beam glow discharge for surface treatment. This simple scheme to reproducibly generate energetic electron beam pulses is expected to find immediate application in materials processing.

This work was partially supported by Grant No. IN063 of the Universidad de Buenos Aires. J. J. Rocca acknowledges the support of the National Science Foundation.

${ }^{1}$ R. Dugdale, J. Mater. Sci. 10, 896 (1975).

${ }^{2}$ H. Iano, J. T. Verdeyen, S. S. Chan, and B. G. Streetman, Appl. Phys. Lett. 39, 622 (1981)

${ }^{3}$ C. Moore, J. J. Rocca, J. Johnson, and G. J. Collins, Appl. Phys. Lett. 43, 290 (1983).

${ }^{4}$ C. Moore, J. Meyer, J. Fukumoto, N. Sluk L. Thompson, J. Knapp, G. J. Collins, and S. Berman, in Silicon on Insulator and Buried Metals in Semiconductors, edited by J. C. Sturm, C. K. Chen, L. Pfeiffer, and P. L. F. Hemment (Materials Research Society, Pittsburgh, 1988), Vol. 107.

${ }^{5}$ F. Ranea-Sandoval, N. Reesor, B. T. Szapiro, C. Murray, and J. J. Rocca, IEEE Trans. Plasma Sci. PS-15, 361 (1987).

${ }^{6}$ N. Mingolo and J. J. Rocca, J. Mater. Res. 7, 1096 (1992).

${ }^{7}$ J. I. Etcheverry, N. Mingolo, J. J. Rocca, and O. E. Martínez, IEEE Trans. Plasma Sci. (in press).

${ }^{8}$ S. A. Lee, L. U. A. Andersen, J. J. Rocca, M. Marconi, and N. D. Reesor, Appl. Phys. Lett. 51, 409 (1987). 